\title{
Age Transformation for Improving Face Recognition Performance
}

\author{
Richa Singh ${ }^{1}$, Mayank Vatsa ${ }^{1}$, Afzel Noore ${ }^{1}$, and Sanjay K. Singh ${ }^{2}$ \\ ${ }^{1}$ West Virginia Univeristy, Morgantown WV 26506, USA \\ richas@csee.wvu.edu, mayankv@csee.wvu.edu, noore@csee.wvu.edu \\ ${ }^{2}$ Purvanchal University, Uttar Pradesh 222001, India \\ sksiet@yahoo.com
}

\begin{abstract}
This paper presents a novel age transformation algorithm to handle the challenge of facial aging in face recognition. The proposed algorithm registers the gallery and probe face images in polar coordinate domain and minimizes the variations in facial features caused due to aging. The efficacy of the proposed age transformation algorithm is validated using 2D log polar Gabor based face recognition algorithm on a face database that comprises of face images with large age progression. Experimental results show that the proposed algorithm significantly improves the verification and identification performance.
\end{abstract}

\section{Introduction}

Human face undergoes significant changes as a person grows older. The facial features vary for every person and are affected by several factors such as exposure to sunlight, inherent genetics, and nutrition. The performance of face recognition systems cannot contend with the dynamics of temporal metamorphosis over a period of time. Law enforcement agencies such as crime and record bureau regularly require matching a probe image with the individuals in the missing person database. In such applications, there may be significant differences between facial features of probe and gallery images due to age variation. For example, if the age of a probe image is 15 years and the gallery image of the same person is of 5 years, existing face recognition algorithms are ineffective and may not yield the desired results.

One approach to handle this challenge is to regularly update the database with recent images or templates. However, this method is not feasible for applications such as border control, homeland security, and missing person identification. To address this issue, researchers have proposed several age simulation and modeling techniques. These techniques model the facial growth over a period of time to minimize the difference between probe and gallery images. Burt and Perrett et al. 1] proposed an age simulation algorithm using shape and texture, and created composite face images for different age groups. They further analyzed and measured the facial cues affected by age variations. Tiddeman [2] proposed wavelet transform based age simulation to prototype the composite face images. Lanitis et al. [3] - 5] proposed statistical models for face simulation. They used 
training images to learn the relationship between coded face representation and actual age of subjects. This relationship was then used to estimate the age of an individual and to reconstruct the face at any age. Gandhi 6] proposed Support Vector Regression to predict the age of frontal faces. He further used the aging function with the image based surface detail transfer method to simulate face image at any younger or older age. Wang et al. 7] obtained the texture and shape information of a face image using PCA and used this information in reconstructing the shape and texture at any particular age. Recently, Ramanathan and Chellappa 8], 9] proposed a craniofacial growth model that characterizes the shape variations in human faces during formative years. They further developed a Bayesian age difference classifier to verify the identity between two images and to estimate the age difference between them.

In this paper, we propose a novel age transformation algorithm to minimize the age difference between two face images. The proposed algorithm is applicable in practical scenarios such as homeland security and missing person database which operates on face images with large age variations as shown in Fig 1 . The proposed age transformation algorithm registers two face images in polar coordinates and minimizes the aging variations. Unlike the conventional method, we transform gallery face images with respect to probe face image and compute the verification and identification performance. Experiments are performed using 2D log polar Gabor transform based face recognition algorithm [10] on a face database of 130 individuals with images of varying age. The proposed algorithm is described in Section 2 and the experimental results are discussed in Section 3.

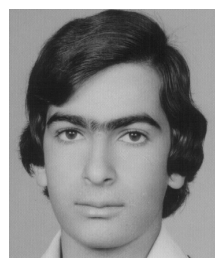

16 years

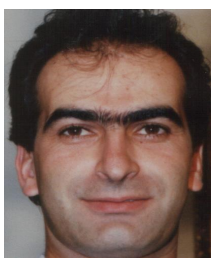

29 years

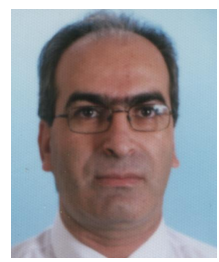

43 years

Fig. 1. Images showing variations in the facial characteristics of an individual at different age [1]

\section{Proposed Registration Based Age Transformation Algorithm}

In this section, we propose the registration based age transformation algorithm which can be used to recognize face images with significant age difference between them. We first explain the algorithm for face verification (1:1 matching) and then extend it to identification (1:N matching). The algorithm is described as follows:

Step 1: Let $F_{G}$ and $F_{P}$ be the detected gallery and probe face images to be matched. $F_{G}(x, y)$ and $F_{P}(x, y)$ are transformed into polar form to obtain 
$F_{G}(r, \theta)$ and $F_{P}(r, \theta)$ respectively. Here, $r$ and $\theta$ are defined with respect to the center coordinate $\left(x_{c}, y_{c}\right)$.

$$
\begin{gathered}
r=\sqrt{\left(x-x_{c}\right)^{2}+\left(y-y_{c}\right)^{2}} \quad 0 \leq r \leq r_{\max } \\
\theta=\tan ^{-1}\left(\frac{y-y_{c}}{x-x_{c}}\right)
\end{gathered}
$$

The coordinates of eyes and mouth are used to form a triangle and the center point of this triangle is chosen as the center point, $\left(x_{c}, y_{c}\right)$, for cartesian to polar conversion. Fig. 2 shows an example of cartesian to polar conversion around the center point. This cartesian to polar conversion eliminates minor variations due to pose and provides robust feature mapping used in the next steps of the algorithm.

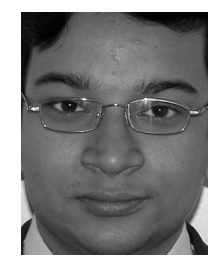

Face Image Cartesian Coordinates

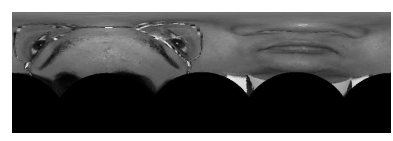

Face Image

Polar Coordinates

Fig. 2. Example of cartesian to polar coordinate conversion

Step 2: The corner features of $F_{G}(r, \theta)$ and $F_{P}(r, \theta)$ are computed using phase congruency based edge and corner detection algorithm [12]. Fig. 3] shows the output of phase congruency based edge and corner detection algorithm on a face image in polar coordinate.

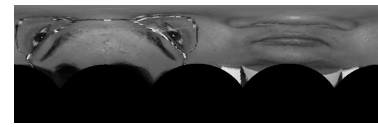

Face Image Polar Coordinates

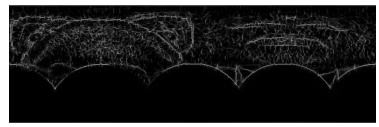

Phase Congruency Map

Fig. 3. Phase congruency map of a polar face image

Step 3: A set of common corner points are selected from the corner features of $F_{G}(r, \theta)$ and $F_{P}(r, \theta)$ using the local correlation technique [13. In the gallery and probe images, a window of size $7 \times 7$ is chosen around the corner points. One to many correlation is then performed on both the gallery and probe images 
and the corner pairs having the maximum correlation output are selected. Also, few corner point pairs could be selected incorrectly. To remove these incorrect pairs, we select only those pairs that have a correlation greater than 0.85 . This technique yields a set of common corner point coordinates which are finally used in the registration based age transformation algorithm.

Step 4: The registration based age transformation is performed on a transformation space, $S$, such that

$$
S=\left[\begin{array}{lll}
a & b & 0 \\
c & d & 0 \\
e & f & 1
\end{array}\right]
$$

where, $a, b, c, d, e, f$ are the transformation parameters for shear, scale, rotation, and translation. We compute the transformation parameter, $S^{\prime}$, by using the common feature point coordinates and the transformation space, $S$,

$$
S^{\prime}=\arg \max _{\{S\}}\left[T\left\{F_{P}(r, \theta), S\left(F_{G}(r, \theta)\right\}\right]\right.
$$

$T\{\cdot\}$ is the feature point registration algorithm described in 14. Thus $F_{G}(r, \theta)$ is registered with respect to $F_{P}(r, \theta)$ using the transformation parameters, $S^{\prime}$.

Step 5: To account for both linear and non-linear variations, we first apply the global transformation followed by the local registration. Global transformation is performed by applying Step 4 on the image to minimize the global variations due to shear, scale, rotation, and translation. Local transformation is then performed on the globally registered images by applying Step 4 in blocks of size $8 \times 8$. This local registration compensates for the non-linear variations in facial features. The registered face images are finally transformed back to cartesian coordinates from polar coordinates.

Fig. 4 shows examples of gallery face image, probe face image, and age difference minimized gallery face image. Once the age difference between gallery and probe face image is minimized, face recognition algorithm can be efficiently applied to verify the identity of the probe image. The proposed algorithm can be easily extended for identification. For identifying any given probe image, first the gallery images are transformed with respect to the probe image and then the probe image is matched with all the transformed gallery images.

\section{$3 \quad$ Experimental Results}

To validate the performance of the proposed registration based age transformation algorithm, we use face aging database which comprises of 1578 images from 130 individuals or classes. The images are obtained partly from the FG-Net database [11 and partly collected by the authors. We divide the face database 

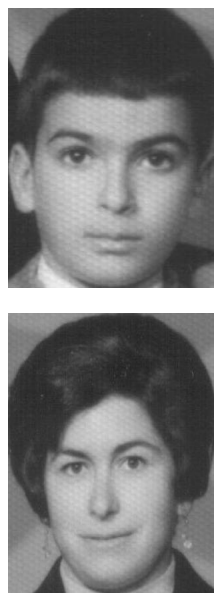

Gallery Image
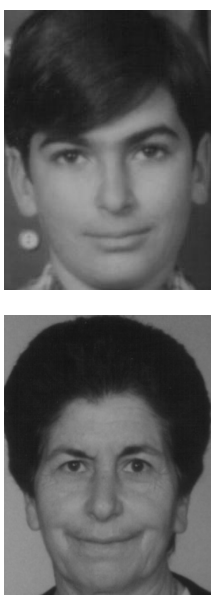

Probe Image
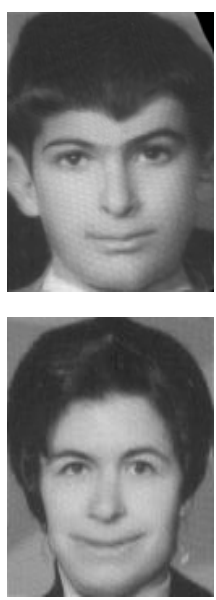

Registered

Gallery Image

Fig. 4. Results of the proposed age transformation algorithm on images from the FGNet face database [1]

into three age groups: (1) 1-18 years, (2) 19-40 years, and (3) beyond 41 years. The database is divided into these three age groups because we observed that face development depends on the age of a person. For example, development in muscles and bone structure cause significant changes in the face during the age of 1-18 years. From 19-40 years, the growth rate is comparatively lower, whereas after 40 years, wrinkles and skin loosening cause major change in facial features and appearance. It is thus very difficult to accurately model an individual's face for very large age variations such as from age 10 to age 60 . Considering these factors, we divide the database into three age groups to evaluate the performance. Details of the images in these three age groups are provided in Table 1. We use one frontal face image per class as the gallery image and the remaining face images as the probe images.

Table 1. Details of the face database used for validation

\begin{tabular}{|l|c|c|c|c|}
\hline \hline $\begin{array}{l}\text { Age } \\
\text { group }\end{array}$ & $\begin{array}{c}\text { Number } \\
\text { of class }\end{array}$ & $\begin{array}{c}\text { Total number } \\
\text { of images }\end{array}$ & $\begin{array}{c}\text { Total number of } \\
\text { images per class }\end{array}$ & $\begin{array}{c}\text { Average age } \\
\text { difference in years }\end{array}$ \\
\hline $1-18$ years & 59 & 605 & $5-16$ & 8 \\
\hline $19-40$ years & 68 & 735 & $8-15$ & 10 \\
\hline Beyond 41 years & 34 & 238 & $4-10$ & 5 \\
\hline
\end{tabular}

We have used 2D log polar Gabor transform based recognition algorithm to match the face images 10. To validate the proposed age transformation algorithm, we compute the performance of the face recognition algorithm both 
with and without the transformation algorithm. The experiments are divided into two parts: face verification and face identification.

\subsection{Face Verification}

To validate the performance of the proposed age transformation algorithm, we first apply the proposed age transformation algorithm on the gallery and probe images. Face recognition algorithm is then applied to match the transformed gallery and probe face images. The performance is computed in terms of verification accuracy at $0.01 \%$ False Accept Rate (FAR). Table 2 shows the verification performance of the face recognition algorithm with and without the proposed age transformation algorithm for the three age groups. For the age group of 1-18 years, an improvement of $37.21 \%$ is observed with the use of the proposed age transformation algorithm. Since the proposed algorithm minimizes the variations by registering gallery and probe images both locally and globally, the performance of face recognition is greatly enhanced. Similarly, for the age group of 19-40 years and beyond 41 years, the performance of face recognition is improved by $23.55 \%$ and $14.49 \%$ respectively.

Table 2. Verification results of the proposed age transformation algorithm. Verification performance is computed at $0.01 \%$ FAR.

\begin{tabular}{|l|c|c|c|}
\hline \hline \multirow{2}{*}{ Age group } & \multicolumn{3}{|c|}{ Verification accuracy (\%) } \\
\cline { 2 - 4 } & $\begin{array}{c}\text { Without registration } \\
\text { based age transformation }\end{array}$ & $\begin{array}{c}\text { With registration based } \\
\text { age transformation }\end{array}$ & $\begin{array}{c}\text { Improvement in } \\
\text { verification accuracy }\end{array}$ \\
\hline $1-18$ years & 21.14 & 58.35 & 37.21 \\
\hline $19-40$ years & 49.87 & 73.42 & 23.55 \\
\hline Beyond 41 years & 72.60 & 87.09 & 14.49 \\
\hline
\end{tabular}

\subsection{Face Identification}

We next evaluate the performance of the proposed algorithm for face identification. Face identification is more challenging than face verification because of the high false accept rate [15. Similar to the face verification experiment, we compute the identification accuracies with and without the proposed registration based age transformation algorithm. Fig. 5 shows the Cumulative Match Characteristic (CMC) plots for this experiment. Rank 1 to 10 identification accuracies for 1-18 years age group shown in Fig. 5(a) clearly indicate that with the proposed age transformation algorithm, the performance of face identification is improved by around $7-18 \%$. For $18-40$ years, an improvement of $18-28 \%$ is observed in the identification performance whereas beyond 41 years an improvement of $7-14 \%$ is observed. The identification experiment thus shows that the proposed age transformation algorithm effectively minimizes the age difference between gallery and probe images and improves the identification performance. 


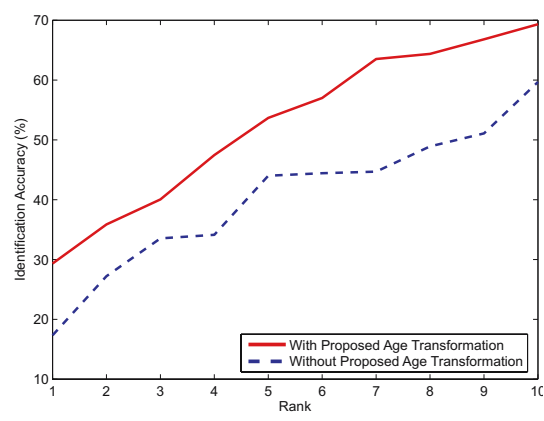

(a)

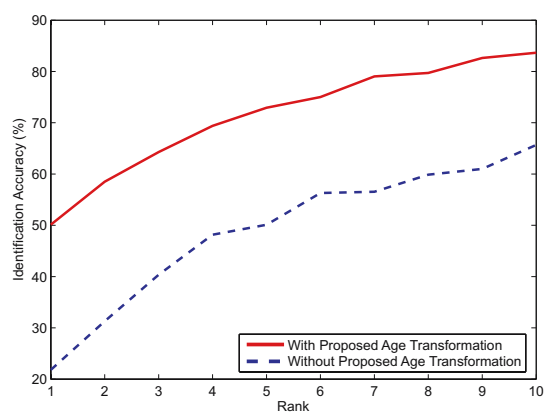

(b)

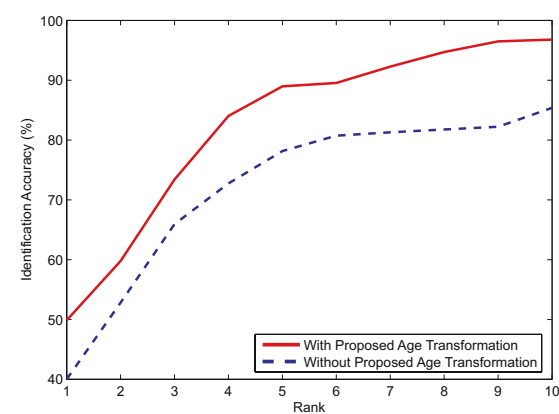

(c)

Fig. 5. CMC plot of the proposed age transformation algorithm for (a) 1-18 years age group, (b) 19-40 years age group, (c) Beyond 41 year age group

\section{Conclusion}

Many law enforcement applications deal with face recognition across age progression. However, existing face recognition algorithms do not perform well in such scenarios. In this paper, we proposed a registration based age transformation algorithm to minimize the age difference between gallery and probe face images. The proposed algorithm registers the gallery and probe face images by transforming them into polar coordinates. The results show significant improvement in the verification and identification performance when the proposed age transformation algorithm is applied. The results also suggest that the proposed algorithm yields better performance for 19-40 years age group and beyond 41 years age group. However, further research is required to improve the recognition performance for 1-18 years age group. Also, additional research is required when dealing with the issue of facial aging along with variations in pose, expression, illumination, and disguise.

\section{Acknowledgment}

Authors would like to acknowledge the FG-NET consortium for providing the FG-Net face database used in this research. 


\section{References}

1. Burt, D.M., Perrett, D.I.: Perception of age in adult caucasian male faces: computer graphic manipulation of shape and colour information. In: Proceedings of Royal Society London, Series B 259, 137-143 (1995)

2. Tiddeman, B., Burt, M., Perrett, D.: Prototyping and transforming facial textures for perception research. IEEE Computer Graphics and Applications 21(5), 42-50 (2001)

3. Lanitis, A., Taylor, C.J., Cootes, T.F.: Toward automatic simulation of aging effects on face images. IEEE Transactions on Pattern Analysis and Machine Intelligence 24(4), 442-455 (2002)

4. Lanitis, A.: On the significance of different facial parts for automatic age estimation. In: Proceedings of International Conference on Digital Signal Processing, vol. 2, pp. 1027-1030 (2002)

5. Lanitis, A., Draganova, C., Christodoulou, C.: Comparing different classifiers for automatic age estimation. IEEE Transactions on Systems, Man, and Cybernactics 34(1), 621-628 (2004)

6. Gandhi, M.: A method for automatic synthesis of aged human facial images, Masters Thesis, Department of Electrical and Computer Engineering, McGill University (2004)

7. Wang, J., Shang, Y., Su, G., Lin, X.: Age simulation for face recognition. In: Proceedings of International Conference on Pattern Recognition, pp. 913-916 (2006)

8. Ramanathan, N., Chellappa, R.: Face verification across age progression. IEEE Transactions on Image Processing 15(11), 3349-3362 (2006)

9. Ramanathan, N., Chellappa, R.: Modeling age progression in young faces. Proceedings of IEEE Computer Vision and Pattern Recognition 1, 387-394 (2006)

10. Singh, R., Vatsa, M., Noore, A.: Face recognition with disguise and single gallery images. Image and Vision Computing (2007)

11. FG-Net Aging Database, http://www.fgnet.rsunit.com/

12. Kovesi, P.D.: Image features from phase congruency, Videre: Journal of Computer Vision Research, MIT Press 1(3) (1999)

13. Singh, R., Vatsa, M., Noore, A.: Improving verification accuracy by synthesis of locally enhanced biometric images and deformable model. Signal Processing 87(11), 2746-2764 (2007)

14. Gonzalez, R.C., Woods, R.E.: Digital image processing, 2nd edn. Prentice Hall, Englewood Cliffs (2002)

15. Li, S.Z., Jain, A.K.: Handbook of face recognition. Springer, Heidelberg (2005) 